\title{
Feedback Finite-Time Stabilization of Impulsive Linear Systems over Piecewise Quadratic Domains
}

\author{
Jing Huang, ${ }^{1}$ Jianxing $\mathrm{Li}^{1}{ }^{1}$ Linshan $\mathrm{Bu}^{2}{ }^{2}$ and Honglei $\mathrm{Xu}{ }^{2}{ }^{2}$ \\ ${ }^{1}$ School of Information Science and Engineering, Fujian University of Technology, Fuzhou, Fujian 350118, China \\ ${ }^{2}$ School of Electrical Engineering, Computing and Mathematical Sciences, Curtin University, Perth, \\ Western Australia 6845, Australia \\ Correspondence should be addressed to Honglei Xu; h.xu@curtin.edu.au
}

Received 17 July 2020; Accepted 17 August 2021; Published 7 September 2021

Academic Editor: Oh-Min Kwon

Copyright (C) 2021 Jing Huang et al. This is an open access article distributed under the Creative Commons Attribution License, which permits unrestricted use, distribution, and reproduction in any medium, provided the original work is properly cited.

\begin{abstract}
This paper investigates the state feedback stabilization problem for a class of impulsive linear time-varying systems over specified time intervals and piecewise quadratic domains (PQDs). First, concepts related to finite-time stability and PQDs are given. Second, finite-time stability analysis over PQDs is implemented, and a variety of stability conditions involving differential linear matrix inequalities are investigated. Then, computationally tractable stability conditions are established for the control design. Finally, an illustrative example is presented to show the effectiveness of the designed state feedback control.
\end{abstract}

\section{Introduction}

Finite-time stability and stabilization are of importance in the applied mathematics and control fields and become a growing cross-disciplinary research area in the past decades. They can be found useful in a variety of applications; for example, when a rocket is launched, it should be controlled to stay in a specified region after a given time interval. Other practical applications include ATM networks [1], neural networks [2], and car suspension systems [3].

In this paper, we are interested in the finite-time stability and stabilization problems of impulsive linear systems in the quantitative sense. The system trajectory evolves in restrained regions during a specified interval of time. The concept of finite-time stability is different from that in the qualitative sense $[4,5]$, which emphasizes that the asymptotically stable system is capable to reach the equilibrium at the settling time. Lots of research results about Lyapunov stability for dynamical systems with impulsive effects have been developed (see, e.g., [6-9] and the references therein). Impulsive synchronization and control problems have attracted much research interest as well [10-16]. Furthermore, a variety of finite-time stability and stabilization problems are investigated for linear time-varying systems and impulsive linear systems $[3,17,18]$. The initial domain $\mathscr{X}_{0}$ and the trajectory domains $\mathscr{X}_{t}$ are usually in the form of ellipsoids and polytopes [3, 19]. Recently, generalized piecewise quadratic domains are proposed for the initial and trajectory domains and stability conditions with less conservatism has been established in [20] and also reviewed in a recent review paper [21]. However, it should be worth noting that the existing stabilization and control methods such as those in $[2,3,17,18]$ are only suitable to ellipsoidal initial and trajectory domains and cannot be applied to the generalized piecewise quadratic domains, which motivates our research of this paper.

This paper investigates the state feedback finite-time stabilization problem for an impulsive linear system. Several sufficient conditions for finite-time stability are derived, and a state feedback control is designed. Comparing with previous work in $[18,19,22]$, this paper has the following main contributions: (1) notions of piecewise quadratic functions and piecewise quadratic domains have been extended to impulsive linear time-varying systems; (2) computationally tractable sufficient conditions for finite-time stability with PQDs are established; and (3) efficient state feedback control to stabilize impulsive linear systems with respect to PQDs is designed. 
The remainder of this paper is as follows. Section 2 presents the impulsive linear system model and preliminary concepts. Section 3 develops sufficient conditions for finitetime stability with PQDs. These stability results involve several computational efficient conditions to design state feedback control. In the next Section 4, a numerical example is given to demonstrate the obtained results. Finally, a conclusion is provided in Section 5 .

1.1. Notations. Let $\mathbb{R}^{+}$denote a set of nonnegative real numbers and $\mathbb{N}^{+}$a set of positive integers. Let $\mathbb{R}^{n}$ be the $n$-dimensional Euclidean space, and $\Omega=[0, T], T>0$, be the time interval. Let $A^{\top}$ denote the transpose of $A$ and $I$ the identity matrix with an appropriate dimension. Let $*$ be the symmetric component of a matrix. The matrix $A \geq 0(A>0)$ is positive semidefinite (positive definite) if $x^{\top} A x \geq 0$ $\left(x^{\top} A x>0\right)$ for all $x \in \mathbb{R}^{n} . A \geq B$ is equivalent to $A-B \geq 0$. For a set $S_{p}=\left\{x_{1}, x_{2}, \ldots, x_{p}\right\} \subseteq \mathbb{R}^{n}$, let cone $\left(S_{p}\right)$ denote its conical hull, i.e., cone $\left(S_{p}\right)=\left\{x \mid x=\sum_{i=1}^{p} \alpha_{i} x_{i}, \alpha_{i} \geq 0\right\}$. Let $\operatorname{Ner}(S)$ denote the set of normalized extremal rays generating $S_{q}$, where $\operatorname{Ner}(S)=\left\{\bar{x}_{1}, \ldots, \bar{x}_{q}\right\}$ with $\left\|\bar{x}_{i}\right\|_{2}=1$, $i=1, \ldots, q \leq p$. For a piecewise continuous matrix-valued (or vector-valued) function $F(\cdot)$ over $\Omega$ and a positive real number $\varepsilon$, let us denote $F^{-}(t)=\lim _{\varepsilon \longrightarrow 0} F(t-\varepsilon)$ and $F^{+}(t)=\lim _{\varepsilon \longrightarrow 0} F(t+\varepsilon)$.

\section{Problem Statement}

Consider the following impulsive linear system with timedependent impulses:

$$
\begin{cases}\dot{x}(t)=A(t) x(t)+B(t) u(t), & x\left(t_{0}\right)=x_{0}, \quad t \notin l=\left\{t_{1}, t_{2}, \ldots\right\} \subset \Omega, \\ x\left(t^{+}\right)=C(t) x(t), & t \in l, \quad k=1,2, \ldots,\end{cases}
$$

where $t \in \mathbb{R}^{+}$is the time, $x(t) \in \mathbb{R}^{n}$ is the state satisfying $x\left(t^{-}\right)=\lim _{t \longrightarrow 0-} x(t)=x(t)$, and $u(t) \in \mathbb{R}^{m}$ is the control. Moreover, $A(t), B(t)$, and $C(t)$ are given matrix-valued functions with appropriate dimensions. Without loss of generally, we assume that there exists a unique solution of equation (1).

Let $U_{i}^{0}(i=1,2, \ldots, v)$ and $U_{j}(j=1,2, \ldots, u)$ be the collections of cones satisfying the following conditions: (1) the dimensions of $U_{i}^{0}$ and $U_{j}$ are equal to $n$; (2) both the union of $U_{i}^{0}, i=1, \ldots, v$, and the union of $U_{j}, j=1, \ldots, u$, can cover the state space $\mathbb{R}^{n}$, i.e., $\cup_{i=1}^{v} U_{i}^{0}=\cup_{j=1}^{u} U_{j}=\mathbb{R}^{n}$; and (3) $\operatorname{In}\left\{U_{p}^{0}\right\} \cap \operatorname{In}\left\{U_{q}^{0}\right\}=\varnothing$ and $\operatorname{In}\left\{U_{p}\right\} \cap \operatorname{In}\left\{U_{q}\right\}=\varnothing$ for all $p \neq q$, where $\operatorname{In}\{\cdot\}$ is the interior operator. We denote by $\Theta\left(U_{p}, U_{q}\right)$ the cone's intersection $U_{p} \cap U_{q}$ and $\operatorname{Ner}(S)=$ $\left\{\widehat{x}_{1}, \ldots, \hat{x}_{q}\right\}$ normalized extremal rays generating $S$ where $\hat{x}_{i 2}=1, i=1, \ldots, q$. First, we need the following concepts on piecewise quadratic domains and finite-time stability over PQDs, which have been defined in $[3,20]$.

Definition 1 (piecewise quadratic functions (PQFs)). A time-varying positive definite quadratic function

$$
P_{P}(x, t)=x^{\top} P_{i}(t) x, \quad \forall x \in U_{i} \text { with } i=1, \ldots, v,
$$

is said to be a piecewise quadratic function over a conical partition $P=\left\{U_{1}, U_{2}, \ldots, U_{v}\right\}$ of $\mathbb{R}^{n}$, where $P_{i} \in \mathbb{R}^{n \times n}, i=1$, $\ldots, v$, are symmetric positive definite matrices in the cone $U_{i}$.

Definition 2 (piecewise quadratic domains (PQDs)). A compact domain whose boundary is the unitary level curve of the piecewise quadratic function $P_{P}(t, x)$ is said to be a piecewise quadratic domain (PQD) over a conical partition $P=\left\{U_{1}, U_{2}, \ldots, U_{v}\right\}$ of $R^{n}$, i.e.,

$$
\begin{aligned}
\mathscr{X}_{P_{P}}(t) & :=\left\{x: P_{P}(t, x) \leq 1\right\}, \\
& =\left\{x: x^{\top} P_{i}(t) x \leq 1, x \in U_{i}, i=1,2, \ldots, v\right\} .
\end{aligned}
$$

Remark 1. Traditionally, both the initial domain and the trajectory domain are given in the form of the standard weighted quadratic norm (i.e., they are in ellipsoidal shapes). These restrictions will be much convenient to introduce quadratic Lyapunov functions to investigate the finite-time stability and stabilization problems. However, the obtained results are not suitable for polytopic domain cases. Piecewise quadratic domains not only are expressed as the class of ellipsoids but also are regarded as the generalization of polytopic domains. They can be applied to model initial and trajectory domains of different forms in many practical applications, such as those in mass-spring-friction systems and electrical circuits $[3,20]$.

Definition 3 (finite-time stability with PQDs). Given two sets $\mathscr{X}_{0}$ and $\mathscr{X}(t), 0 \in \mathscr{X}_{0}$, the system equation (1) is said to be finite-time stable with respect to $\left(\Omega, \mathscr{X}_{0}, \mathscr{X}(t)\right)$ if

$$
x_{0} \in \mathscr{X}_{0} \text { implies } x(t) \in \mathscr{X}(t) \text { for } t \in \Omega \text {, }
$$

where the initial and trajectory domains are described as $\mathscr{X}_{0}=$ $\left\{x \in \mathbb{R}^{n} \mid x_{0}^{\top} R_{i} x_{0} \leq 1, x_{0} \in U_{i}^{0}, i=1,2, \ldots, u\right\} \subseteq \mathscr{X}\left(t_{0}\right)$ and $\mathscr{X}_{t}$ $=\left\{x^{\top} Q_{j}(t) x \leq 1, x \in U_{j}, j=1,2, \ldots, v\right\}$ over conical partitions $P_{0}=\left\{U_{1}^{0}, U_{2}^{0}, \ldots, U_{u}^{0}\right\}$ and $P=\left\{U_{1}, U_{2}, \ldots, U_{v}\right\}$.

This paper aims to design a feedback controller $u(t)=$ $F x(t), F \in \mathbb{R}^{m \times n}$ such that the controlled impulsive linear system equation (1) ensures the finite-time stability with PQDs. Now, we need to introduce the following lemma.

Lemma 1 (see $[20,22])$. For a piecewise quadratic function $P_{P}(t, x)$ over the given conical partition $P$, we denote by $v_{P}=$ $\left\{\bar{x}_{1}, \bar{x}_{2}, \ldots, \bar{x}_{q}\right\}$ the set of generating rays. The piecewise quadratic function $P_{P}(x, t)$ is continuous if and only if

$$
\begin{aligned}
& \bar{x}_{h}^{\top} P_{i}(t) \bar{x}_{h}=\bar{x}_{h}^{\top} P_{j}(t) \bar{x}_{h}, \\
& \bar{x}_{h}^{\top} P_{i}(t) \bar{x}_{l}=\bar{x}_{h}^{\top} P_{j}(t) \bar{x}_{l},
\end{aligned}
$$

for all $\bar{x}_{h}, \bar{x}_{l} \in \operatorname{Ner}\left\{\Theta\left(U_{i}, U_{j}\right)\right\}$, where $\Theta\left(U_{i}, U_{j}\right)=U_{i} \cap U_{j}$.

\section{Main Results}

In this section, we establish several sufficient conditions of finite-time stability with PQDs for the impulsive linear system equation (1). For simplicity, we consider the initial 
set $\mathscr{X}_{0}$ and the time-varying set $\mathscr{X}_{t}$ to be piecewise quadratic domains over the conical partition $P=\left\{U_{i}\right\}, i=1,2, \ldots, u$. Then, when the control $u(t)=0$, we have the following sufficient conditions for finite-time stability with PQDs.

Theorem 1. The system equation (1) is finite-time stable with respect to $\left(\Omega, \mathscr{X}_{0}, \mathscr{X}_{t}\right)$, where the sets $\mathscr{X}_{0}$ and $\mathscr{X}_{t}$ are the given $P Q D s$, if there exist a positive monotone increasing function $\rho(\cdot)$ and a piecewise Lyapunov-like function $V(t, x)$ such that

$$
\begin{gathered}
\frac{\partial V(t, x)}{\partial t}+\frac{\partial V(t, x)}{\partial x} A(t) x(t)<0, \quad t \notin l, \\
V\left(t^{+}, x\right)<V(t, x), \quad t \in l, \\
\rho\left(x^{\top} Q_{i}(t) x\right) \leq V(t, x), \\
V\left(t_{0}, x_{0}\right)<\rho\left(x_{0}^{\top} R_{i} x_{0}\right),
\end{gathered}
$$

for $t \in \Omega$ and $x \in U_{i}$ with $i=1, \ldots, v$.

Proof. We choose $x_{0}$ satisfying $x_{0}^{\top} R_{i} x_{0} \leq 1$ and denote by $x\left(t, x_{0}\right)$ the solution of the system equation (1). Next, we consider the case $t \in\left(t_{k}, t_{k+1}\right]$. Based on the condition equation (8), we have

$$
x^{\top} Q_{i}(t) x \leq \rho^{-1}(V(t, x)) .
$$

Moreover, the condition equation (6) ensures that $V(t, x)$ will decrease along the solution of the system equation (1), and hence we have $V(t, x) \leq V\left(t_{k}^{+}, x\left(t_{k}^{+}\right)\right)$, for $t \in\left(t_{k}, t_{k+1}\right]$. In view of equation (7), we say $V(t, x) \leq V\left(t_{k}, x\left(t_{k}\right)\right)$ will be satisfied. Repeatedly using equations (6) and (7), we get

$$
V(t, x)<V\left(t_{0}, x_{0}\right) .
$$

Hence, it follows from equations (9) and (10) and the fact that $\rho(\cdot)$ is positive monotone increasing that

$$
x^{\top} Q_{i}(t) x<\rho^{-1}\left(V\left(t_{0}, x_{0}\right)\right) .
$$

Finally, using equation (8) and the fact $x_{0}^{\top} R_{i} x_{0} \leq 1$, we obtain $x^{\top} Q_{i}(t) x<1$. It completes the proof.

We choose a positive definite monotone increasing function $\rho(\eta)=\eta$ where $\eta \in \mathbb{R}^{+}$and a piecewise quadratic Lyapunov-like function over the above conical partition $P$ as

$$
V_{X}(t, x)=x^{\top} P_{i}(t) x, \quad \forall x \in U_{i}, \text { with } i=1, \ldots, v,
$$

where $P_{i} \in \mathbb{R}^{n \times n}, i=1, \ldots, v$, are symmetric matrices. Then, a sufficient condition for the finite-time stability of impulsive linear system equation (1) can be given as follows.

Theorem 2. The system equation (1) is finite-time stable with respect to $\left(\Omega, \mathscr{X}_{0}, \mathscr{X}_{t}\right)$, where the sets $\mathscr{X}_{0}$ and $\mathscr{X}_{t}$ are the given $P Q D s$, if there exist piecewise continuously differentiable matrix-valued functions $P_{i}(t) \in \mathbb{R}^{n \times n}$ such that

$$
\begin{gathered}
x^{\top}\left(\dot{P}_{i}(t)+A(t)^{\top} P_{i}(t)+P_{i}(t) A(t)\right) x<0, \quad t \notin l, \\
x^{\top} C^{\top}(t) P_{i}^{+}(t) C(t) x<x^{\top} P_{i}(t) x, \quad t \in l, \\
x^{\top}\left(Q_{i}(t)-P_{i}\right) x \leq 0, \quad x_{0}^{\top}\left(P\left(t_{0}\right)-R_{i}\right) x_{0}<0,
\end{gathered}
$$

for $t \in \Omega$ and $x \in U_{i}$ with $i=1, \ldots, v$.

Proof. By choosing $V_{X}(t, x)=x^{\top} P_{i}(t) x$, it is straightforwardly derived that equations (11)-(13) can be guaranteed by equations (6)-(8). Then, by Theorem 1 , we ensure that the system equation (1) is finite-time stable with respect to $\left(\Omega, \mathscr{X}_{0}, \mathscr{X}_{t}\right)$.

Sufficient conditions equations (11)-(13) in Theorem 2 are only theoretically useful because of the existence of the infinite number of differential linear matrix inequalities. Applying $S$-Procedure arguments and using the state feedback control $u(t)=F x(t)$, we can derive the following computationally tractable sufficient conditions.

Corollary 1. The system equation (1) is finite-time stabilizable with respect to $\left(\Omega, \mathscr{X}_{0}, \mathscr{X}_{t}\right)$ under the feedback control law $u(t)=F x(t)$, where the sets $\mathscr{X}_{0}$ and $\mathscr{X}_{t}$ are the given $P Q D$ s if there exist positive numbers $b_{i, l}$, positive real-valued functions $c_{i, l}(t), z_{i, l}(t)$, and matrices $H_{i, l}$, satisfying $x^{\top} H_{i, l} x \leq 0, \forall x \in S_{i}, i=1, \ldots, v, l=1, \ldots, s$ such that there exist positive piecewise continuously differentiable matrixvalued functions $P_{i}(t) \in \mathbb{R}^{n \times n}$, such that the following conditions containing differential linear matrix inequalities are satisfied:

$$
\begin{aligned}
\dot{P}_{i}(t) & +(A(t)+B(t) F]^{\top} P_{i}(t)+P_{i}(t)(A(t)+B(t) F) \\
& -\sum_{l=1}^{s} c_{i, l}(t) Q_{i, l}<0, \quad t \notin l,
\end{aligned}
$$

$$
\begin{gathered}
C^{\top}(t) P_{i}^{+}(t) C(t)<P_{i}(t), \quad t \in l, \\
P_{i}(t)-Q_{i}(t)+\sum_{l=1}^{s} z_{i, l}(t) H_{i, l} \geq 0, \\
P_{i}(0)-R_{i}-\sum_{l=1}^{s} b_{i, l} H_{i, l}<0, \\
\bar{x}_{l}^{\top} P_{i}(t) \bar{x}_{l}=\bar{x}_{l}^{\top} P_{j}(t) \bar{x}_{l}, \quad \forall \bar{x}_{l} \in \operatorname{Ner}\left\{U_{i} \cap U_{j}\right\}, \\
\bar{x}_{h}^{\top} P_{i}(t) \bar{x}_{l}=\bar{x}_{h}^{\top} P_{j}(t) \bar{x}_{l}, \quad \forall \bar{x}_{h}, \bar{x}_{l} \in \operatorname{Ner}\left\{U_{i} \cap U_{j}\right\} .
\end{gathered}
$$

Proof. Using S-Procedure and Theorem 5 of [20], we obtain that the conditions equations (13)-(15) are derived if the conditions equations (16) and (19) are satisfied. Moreover, it follows from Lemma 1 and equations (20) and (21) that the piecewise quadratic Lyapunov function $V_{\mathscr{X}}(t, x)=x^{\top} P_{i}(t) x$ is continuous. Thus, by Theorem 2 , the conclusion of this theorem is obtained. 


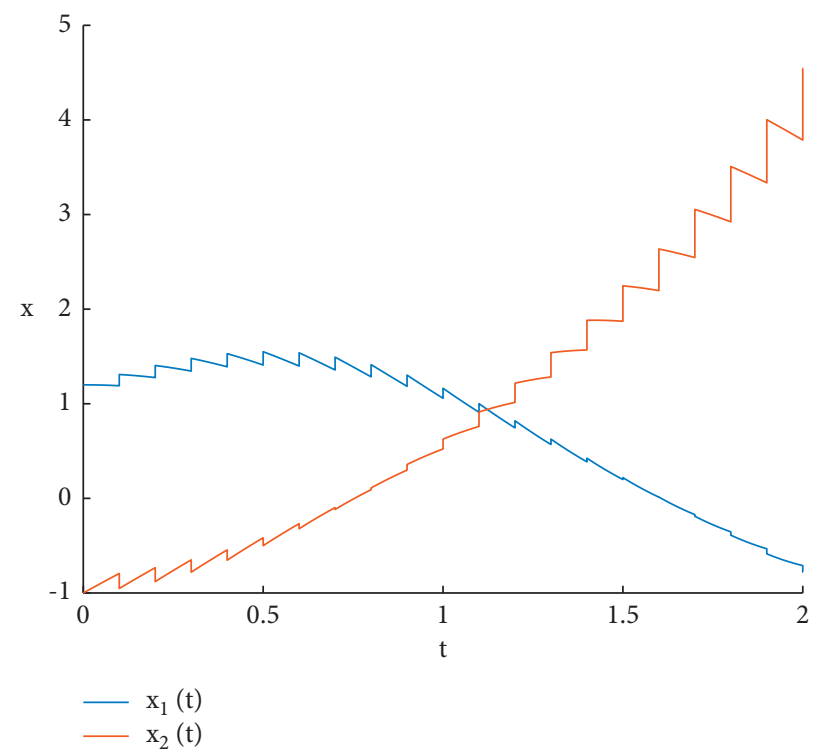

Figure 1: State trajectories $x_{1}(t)$ and $x_{2}(t)$ of the uncontrolled impulsive linear system equation (22) with $0.1 \mathrm{~s}$ equidistant impulsive intervals.

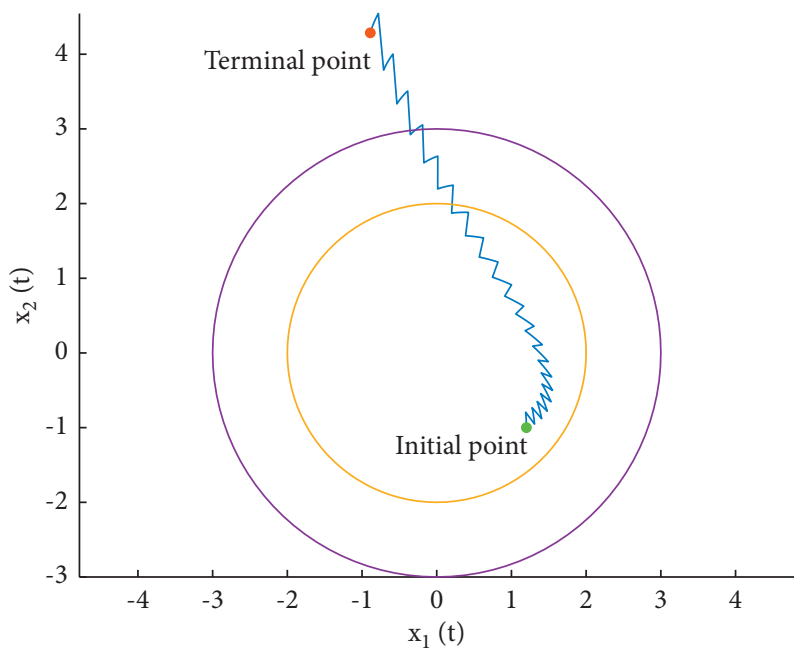

Figure 2: Phase portrait of the uncontrolled impulsive linear system equation (22) with $0.1 \mathrm{~s}$ equidistant impulsive intervals.

Remark 2. In Corollary 1, solving differential linear matrix inequalities and deciding the conical partitions are two main steps to influence the computational complexity. In the former step, more accurate approximation will increase the computational complexity, and in the later step, a greater number of the conical partitions will cause the computational burden as well.

\section{An Illustrative Example}

In this section, we give an example to demonstrate the effectiveness of the proposed conditions. Let us consider the following impulsive linear control system:

$$
\begin{cases}\dot{x}(t)=\left[\begin{array}{cc}
-3 t & 1 \\
1.5 & t
\end{array}\right] x(t)+\left[\begin{array}{ll}
1.5 & 2 \\
1.6 & 1
\end{array}\right] u(t), & t \notin l=\{0.1, \ldots, 0.1 k, \ldots\}, \\
x\left(t^{+}\right)=\left[\begin{array}{cc}
1.1 & 0 \\
0 & 1.2
\end{array}\right] x\left(t^{-}\right), & t \in l, k \in \mathbb{N}^{+},\end{cases}
$$




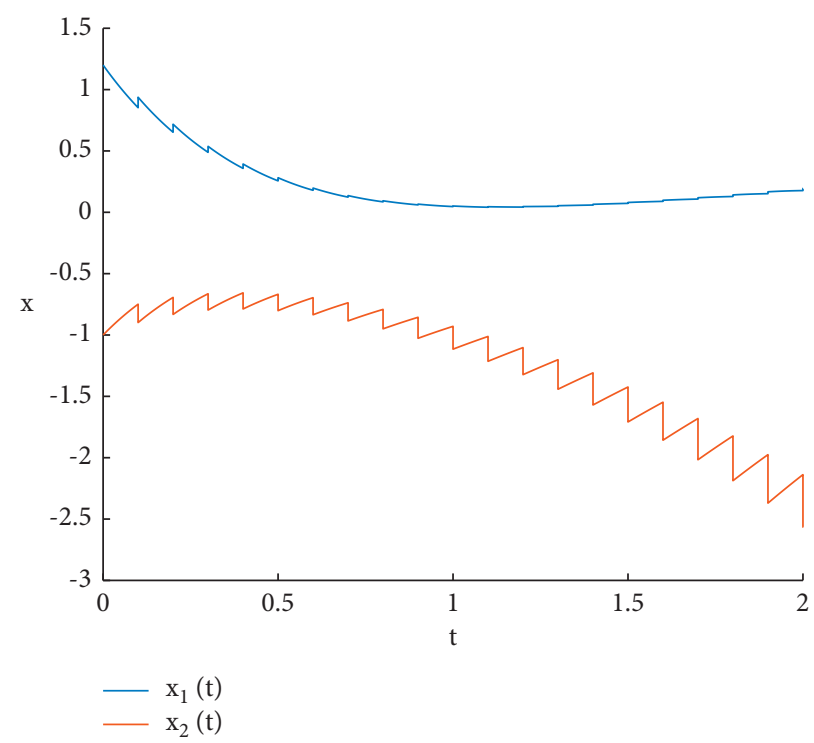

FIGURE 3: State trajectories $x_{1}(t)$ and $x_{2}(t)$ of the impulsive linear control system equation (22) with $0.1 \mathrm{~s}$ equidistant impulsive intervals.

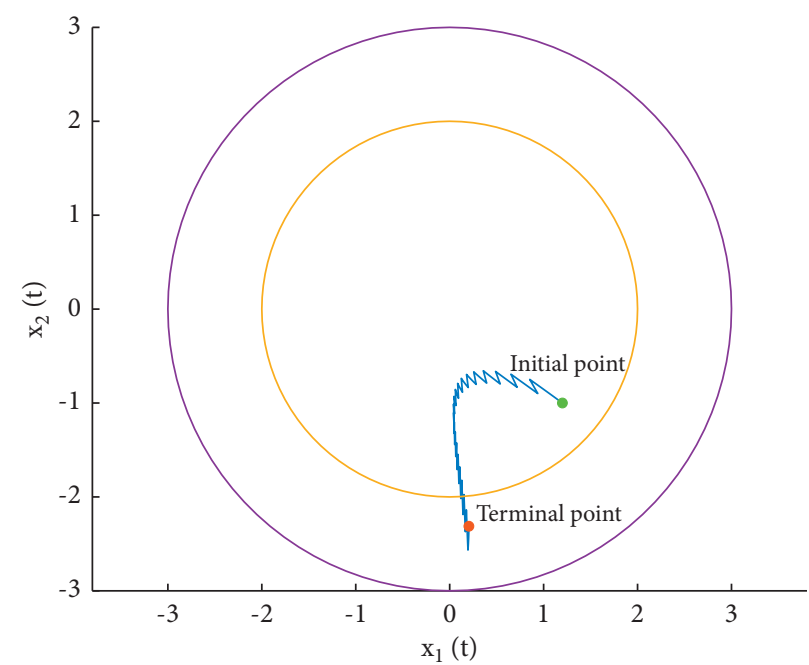

FIgURE 4: Phase portrait of the impulsive linear control system equation (22) with $0.1 \mathrm{~s}$ equidistant impulsive intervals.

with $t \in \Omega=[0,2]$ and the initial and trajectory domains

$$
\mathscr{X}_{0}=\left\{x: x^{\top} x<4\right\} \text {, and } \mathscr{X}_{t}=\left\{x: x^{\top} x<9\right\} .
$$

When the state feedback control $u(t)$ is not implemented, the state trajectories and phase portrait of the impulsive linear system equation (10) with $0.1 \mathrm{~s}$ equidistant impulsive intervals are seen in Figures 1 and 2. From them, we can see that the state has been outside the set $X_{t}$ at $T=2$, and hence the impulsive linear system equation (22) is not finite-time stable with respect to $\left(\Omega, \mathscr{X}_{0}, \mathscr{X}_{t}\right)$. Since in this example both initial and trajectory domains are ellipsoidal, the piecewise quadratic Lyapunov function will be continuous everywhere. Hence, equations (20) and (21) will be guaranteed straightforwardly. By using conditions equations (16)-(19), we can obtain a feasible solution for the state feedback matrix:

$$
F=\left(\begin{array}{cc}
3.1765 & -0.9412 \\
-3.8824 & 0.9059
\end{array}\right) .
$$

Then, applying $u(t)=F x(t)$ to equation (22), we simulate the state trajectories and phase portrait of the impulsive linear system equation (10) with $0.1 \mathrm{~s}$ equidistant impulsive interval in Figures 3 and 4, which show that the impulsive linear control system equation (22) is finite-time stable with respect to the given $\left(\Omega, \mathscr{X}_{0}, \mathscr{X}_{t}\right)$. So, the designed state feedback finite-time stabilizing controller is effective.

\section{Conclusion}

This paper has investigated the feedback finite-time stabilizing control problem for impulsive linear systems with respect to PQDs. First, the concepts of piecewise quadratic 
functions and piecewise quadratic domains are provided. Finite-time stability in the quantitative sense is investigated. Then, sufficient conditions of finite-time stability with PQDs for impulsive linear systems are established. Based on these stability criteria, computationally tractable conditions to design state feedback control for the impulsive linear systems are derived. A numerical example is finally given to demonstrate the usefulness of the designed state feedback control.

\section{Data Availability}

The data used to support the findings of this study are available from the corresponding author upon request.

\section{Conflicts of Interest}

The authors declare that there are no conflicts of interest.

\section{Acknowledgments}

This work was supported in part by the Australian Research Council (DP160102819), the Research Program of Fujian University of Technology (GY-Z18002), and the Open Fund Project of Key Scientific Research and Innovation Platform of Fujian University of Technology (2018).

\section{References}

[1] F. Amato, M. Ariola, C. T. Abdallah, and C. Cosentino, "Application of finite-time stability concepts to the control of ATM networks," in Proceedings of the Annual Allerton Conference on Communication Control and Computing, pp. 1071-1079, Monticello, IL, USA, October 2002.

[2] P. Niamsup, K. Ratchagit, and V. N. Phat, "Novel criteria for finite-time stabilization and guaranteed cost control of delayed neural networks," Neurocomputing, vol. 160, pp. 281-286, 2015.

[3] F. Amato, R. Ambrosino, M. Ariola, C. Cosentino, and G. De Tommasi, Finite-Time Stability and Control, SpringerVerlag, Berlin, Germany, 2014.

[4] S. P. Bhat and D. S. Bernstein, "Finite-time stability of continuous autonomous systems," SIAM Journal on Control and Optimization, vol. 38, no. 3, pp. 751-766, 2000.

[5] S. P. Bhat and D. S. Bernstein, "Geometric homogeneity with applications to finite-time stability," Mathematics of Control, Signals, and Systems, vol. 17, no. 2, pp. 101-127, 2005.

[6] X. J. Ding and H. L. Xu, "Robust stability and stabilization of a class of impulsive switched systems," Dynamics of Continuous Discrete and Impulsive Systems: Series B; Applications and Algorithms, vol. 2, pp. 795-798, 2005.

[7] X. Li and R. Rakkiyappan, "Impulsive controller design for exponential synchronization of chaotic neural networks with mixed delays," Communications in Nonlinear Science and Numerical Simulation, vol. 18, no. 6, pp. 1515-1523, 2013.

[8] H. Xu and K. Lay Teo, " $H_{\infty}$ optimal stabilization of a class of uncertain impulsive systems: an LMI approach," Journal of Industrial and Management Optimization, vol. 5, no. 1, pp. 153-159, 2009.

[9] H. Xu and K. L. Teo, "Stabilizability of discrete chaotic systems via unified impulsive control," Physics Letters A, vol. 374, no. 2, pp. 235-240, 2009.
[10] B. Liu, X. Liu, G. Chen, and H. Wang, "Robust impulsive synchronization of uncertain dynamical networks," IEEE Transactions on Circuits and Systems I: Regular Papers, vol. 52, no. 7, pp. 1431-1441, 2005.

[11] X. Liu, Y. Liu, and L. T. Kok, "Stability analysis of impulsive control systems," Mathematical and Computer Modelling, vol. 37, no. 12-13, pp. 1357-1370, 2003.

[12] L. Li, X. Shi, and J. Liang, "Synchronization of impulsive coupled complex-valued neural networks with delay: the matrix measure method," Neural Networks, vol. 117, pp. 285-294, 2019.

[13] M. Li, H. Chen, and X. Li, "Synchronization analysis of complex dynamical networks subject to delayed impulsive disturbances," Complexity, vol. 2020, Article ID 5285046, 12 pages, 2020.

[14] G. Mu, L. Li, and X. Li, "Quasi-bipartite synchronization of signed delayed neural networks under impulsive effects," Neural Networks, vol. 129, pp. 31-42, 2020.

[15] X. Xie, X. Liu, H. Xu, X. Luo, and G. Liu, "Synchronization of coupled reaction-diffusion neural networks: delay-dependent pinning impulsive control," Communications in Nonlinear Science and Numerical Simulation, vol. 79, Article ID 104905, 2019.

[16] X. Xie, X. Liu, and H. Xu, "Synchronization of delayed coupled switched neural networks: mode-dependent average impulsive interval," Neurocomputing, vol. 365, pp. 261-272, 2019.

[17] F. Amato, M. Ariola, M. Carbone, and C. Cosentino, "Finitetime control of linear systems: a survey," in Current Trends in Nonlinear Systems and Control, L. Menini, L. Zaccarian, and C. T. Abdallah, Eds., pp. 195-213, Springer, New York, NY, USA, 2006.

[18] F. Amato, R. Ambrosino, M. Ariola, and G. De Tommasi, "Robust finite-time stability of impulsive dynamical linear systems subject to norm-bounded uncertainties," International Journal of Robust and Nonlinear Control, vol. 21, no. 10, pp. 1080-1092, 2011.

[19] F. Amato, R. Ambrosino, M. Ariola, and C. Cosentino, "Finite-time stability of linear time-varying systems with jumps," Automatica, vol. 45, no. 5, pp. 1354-1358, 2009.

[20] R. Ambrosino, E. Garone, M. Ariola, and F. Amato, "Piecewise quadratic functions for finite-time stability analysis," in Proceedings of the 51st IEEE Conference on Decision and Control (CDC), pp. 6535-6540, IEEE, Maui, HI, USA, December 2012.

[21] H. Xu, "Finite-time stability analysis: a tutorial survey," Complexity, vol. 2020, Article ID 1941636, 12 pages, 2020.

[22] R. Ambrosino and E. Garone, "Robust stability of linear uncertain systems through piecewise quadratic lyapunov functions defined over conical partitions," in Proceedings of the 51st IEEE Conference on Decision and Control (CDC), pp. 2872-2877, IEEE, Maui, HI, USA, December 2012. 